\title{
Síndrome de Sjögren e a prática da odontologia: revisão do conhecimento atual
}

\author{
Sjögren's Syndrome and dental practice: \\ a review of current knowledge \\ Armiliana Soares NASCIMENTO \\ Mestranda do Programa de Pós-Graduação \\ em Odontologia pela Universidade Estadual da Paraíba (UEPB). \\ Yêska Paola Costa AGUiar \\ Mestranda do Programa de Pós-Graduação \\ em Odontologia pela Universidade Estadual da Paraíba (UEPB). \\ Roniery Oliveira COSTA \\ Mestrando do Programa de Pós-Graduação \\ em Odontologia pela Universidade Estadual da Paraíba (UEPB). \\ Maria Helena Chaves de Vasconcelos Catão \\ Doutora em Laser em Odontologia/UFBA e professora \\ do Programa em Pós-Graduação da UEPB. \\ Luciana de Barros Correia FONTES \\ Doutora em Laser em Odontologia/UFBA e professora \\ do Programa em Pós-Graduação da UEPB.
}

\section{RESUMO}

Existe uma preocupação crescente quanto aos fatores implicados na etiologia da síndrome de Sjögren, assim como os métodos de diagnóstico e de tratamento, com vistas a minimizar o impacto na qualidade de vida de seus portadores. Nesse contexto, um cuidado maior tem sido direcionado à possibilidade do acometimento primário na infância, com morbidade associada, e pela subnotificação constatada. $O$ estudo presente busca efetuar uma revisão atualizada da literatura sobre o tema, destacando o papel dos profissionais da odontologia. Para alcançar o objetivo, efetuou-se uma consulta a artigos indexados nas bases de dados Lilacs, Medline e Scielo, adotando-se os seguintes descritores: síndrome de Sjögren, etiologia, diagnóstico, terapêutica e odontologia, no período compreendido entre os anos de 2002 e 2012. Após a análise dos trabalhos consultados, verificou-se que a etiologia continua até então obscura e que os métodos de diagnóstico permanecem praticamente os mesmos, com lacunas de informação sobre as notificações efetuadas durante a assistência em saúde bucal. A reabilitação dos pacientes com síndrome de Sjögren é um desafio complicado, uma vez que o tratamento consiste apenas no alívio da sintomatologia e, por ser administrada no longo prazo, acarreta efeitos secundários adversos.

Palavras-chave: SÍNDROME DE SJÖGREN; DIAGNÓSTICO; TERAPÉUTICA; ODONTOLOGIA.

\begin{abstract}
There is growing concern about the factors involved in the etiology of Sjögren's syndrome, as well as the methods of diagnosis and treatment, in order to minimize the impact on the patients' quality of life. In this context, greater attention has been given to the possibility of primary involvement in childhood, with associated morbidity, due to the observed underreporting. The present study seeks to perform a literature review on the topic, highlighting the role of dental professionals. To achieve the goal we performed a query for articles indexed in Lilacs, Medline and Scielo databases, adopting the following descriptors: Sjögren's Syndrome, etiology, diagnosis, therapeutics and dentistry in the period between the years 2002 and 2012. After the analysis of the studies, it was found that the etiology remains obscure and the diagnostic methods remain largely the same, with gaps of information on the notifications made during oral health care. The rehabilitation of patients with Sjögren's syndrome is a difficult challenge since the only treatment consists in relieving the symptoms and, due to being administered in the long term, it causes adverse side effects to patients.
\end{abstract}

Keywords: SJÖGREN'S SYNDROME, DIAGNOSIS, THERAPEUTICS, DENTISTRY. 


\section{INTRODUÇÃO}

A síndrome de Sjögren (SS) foi descrita pela primeira vez em 1957, caracterizada por ictiose congênita, diplegia de membros inferiores e retardo mental. ${ }^{1}$ A partir de então, vários estudos vêm sendo desenvolvidos no intuito de reconhecer o quadro clínico, histopatológico e suas características imunogenéticas e sorológicas. ${ }^{2}$ Constitui uma doença autoimune inflamatória de curso crônico frequente, na qual o sistema imunológico do paciente ataca as glândulas salivares e lacrimais, ${ }^{3} \mathrm{com}$ prevalência similar à encontrada na artrite reumatoide. Pode estender-se e abranger muitos órgãos ou sistemas. ${ }^{4}$ Nela existem dois tipos reconhecidos: a primária - que não se manifesta associada a outra desordem e se caracteriza pela presença de ceratoconjuntivite seca, xerostomia e anormalidades extraglandulares; e a secundária - na qual o paciente manifesta a síndrome associada a outras doenças autoimunes. ${ }^{5}$

Apesar de apresentar etiologia um tanto quanto obscura, admite-se que diferentes fatores, entre os quais os genéticos e ambientais, sejam possíveis desencadeadores de seu desenvolvimento e curso clínico. Seu diagnóstico é obtido pelo somatório de achados clínicos e laboratoriais, com base na sintomatologia e na associação a outras doenças $^{6}$ e o tratamento consistindo apenas no alívio dos sintomas. ${ }^{7}$

Detectar a SS na infância constitui um processo mais complicado, especialmente pela diversidade da apresentação clínica e da dificuldade em obterem-se dados confiáveis sobre a história; há uma maior frequência de casos subdiagnosticados. Condições diferenciais devem ser consideradas, especialmenteasque promovemaxerostomia, tais como: diabetes, displasia ectodérmica, artrite reumatoide, esclerodermia, lúpus eritematoso sistêmico, sarcoidose, linfoma, infecção por HIV e HTLV. Outras, associadas com o aumento da parótida, também devem ser excluídas: parotidite recorrente juvenil (JRP), sialadenosis, sarcoidose, linfoma, parotidite infecciosa causada por infecções por estreptococos e estafilococos, infecções virais (paramixovírus, vírus de Epstein-Barr, citomegalovírus, e parvovírus), síndrome linfocitose difusa infiltrativa (associada com a infecção pelo HIV) e doenças congênitas raras, como a doença policística parótida. ${ }^{8}$

Sabe-se que a grande maioria dos pacientes com a síndrome de Sjögren apresenta manifestações orais; no entanto, não se estabeleceu ainda um quadro completo dessas características e de suas sequelas. A queixa oral constitui o sintoma inicial em mais da metade dos portadores, havendo uma sequência possível de sinais e sintomas persistentes e de complexidade ampla que determinam um ônus significativo da doença. Minimizar as morbidades orofaciais constitui um desafio. ${ }^{9}$

O início dos sintomas ocorre mais frequentemente durante a meia-idade. Os pacientes apresentam ressecamento da boca e dos olhos; os sintomas incluem fadiga extrema, mialgia, artralgia e sofrimento psíquico. Quanto as manifestações bucais a hipossalivação e o ressecamento da mucosa oral expoem esses pacientes á um alto risco para uma saúde bucal deficiente. Os pacientes também podem experimentar mudanças na composição da saliva, o que pode levar ao aumento da suscetibilidade à cárie dentária, à doença periodontal, à candidíase oral e à ulceração, além de provocar mudanças na sensação gustativa e complicar o uso de próteses dentárias. Em acréscimo, experimentam dificuldade de mastigação e deglutição de alimentos. Transtornos na fala e a possibilidade acentuada de halitose, como resultado de xerostomia, estão associados a constrangimentos em situações sociais. ${ }^{10}$

Diante dos impactos negativos dessa síndrome na qualidade de vida dos acometido 
e das lacunas e controvérsias com relação à etiologia, ao diagnóstico e ao tratamento, objetiva-se, a partir do presente estudo, realizar uma revisão de literatura recente sobre o tema, explorando o estado da arte sobre os pontos supracitados.

\section{REVISÃO DA LITERATURA}

Para facilitar a leitura sobre o assunto em questão, houve uma subdivisão em tópicos. Os artigos selecionados encontram-se indexados nas bases de dados Lilacs, Medline e Scielo, a partir dos descritores síndrome de Sjögren, etiologia, diagnóstico, terapêutica e odontologia, no período compreendido entre os anos 2002 e 2012.

\section{Prevalência e quadro clínico}

Pouco comum em crianças, a síndrome acomete, preferencialmente, adultos entre a quarta e quinta décadas de vida, com prevalência de 9:1 a 10:1 para o sexo feminino em relação ao masculino. A razão para a predominância em mulheres ainda permanece desconhecida, porém existe uma corrente que defende a participação dos hormônios - teoria embasada na deficiência de androgênios, considerando-se que maioria dos pacientes adquirem a doença no período da menopausa e/ou andropausa. ${ }^{11}$ Nikolov e Illei ${ }^{12}$ evidenciaram fatores sexuais predisponentes em virtude de um aumento na proporção androgênio/estrogênio.

Considerada uma doença nova em termos de pesquisas, a SS não é tão rara ao se verificar o número de pessoas acometidas. Existe um relato variado de sua prevalência, entre $0,2 \%$ a $4 \%$ na população mundial, em virtude dos diferentes métodos de diagnóstico empregados. ${ }^{13,14,15}$

Para a população da América do Norte, a SS acomete de $1 \%$ a $4,2 \%$ dos indivíduos, embora a maioria dos casos permaneça sem diagnóstico. ${ }^{16}$ Nos países europeus estima-se uma prevalência de $0,6 \%$ a 3,3\%, com incidência de quatro casos por 100 mil vivos, enquanto que no Brasil, pela ausência de estimativas oficiais ou cientificamente confirmadas sobre sua incidência, não se sabe o número exato de portadores dessa síndrome. Porém existe a referência quanto à maioria dos casos diagnosticados contemplar mulheres na menopausa ou em idade mais avançada. ${ }^{17}$

Com relação às condições clínicas, os pacientes podem apresentar sinais e sintomas oculares e orais, envolvimento da pele, mucosa nasal e vaginal, assim como fadiga, cirrose biliar primária, fibrose pulmonar, linfadenopatia, nefrite intestinal, vasculites e neuropatias periféricas. ${ }^{18}$ Ainda ocorrem registros de ceratonjuntivite seca, xerostomia e edema das glândulas salivares, principalmente da glândula parótida. ${ }^{19}$

Quanto às manifestações orais, frequentemente os acometidos relatam boca seca (necessidade de umedecimento-ingestão de líquidos durante a noite), língua lobulada, lesões de cáries frequentes, comprometimento da qualidade do sono, dificuldade na deglutição de alimentos sólidos, dor na deglutição, aftas e úlceras na mucosa labial, infecções bucais (principalmente candidíase) e aumento no volume da glândula parótida (mais frequente na SS primária). ${ }^{20}$

\section{Etiologia}

De etiologia ainda obscura, a SS apresenta fatores genéticos e ambientais como possíveis desencadeadores do seu desenvolvimento e curso clínico. Acredita-se ser a resposta autoimune na SS, impulsionada por proteínas celulares que atuam como autoantígenos epiteliais, acompanhadas de apoptose celular. ${ }^{21}$

Parte-se do princípio de que a infecção viral possa criar um microambiente 
inflamatório que modifica a disposição dos fragmentos apoptóticos servindo como autoantígenos. ${ }^{22}$ Desse modo, a imunidade mediada por células apresenta um papel importante na SS por levar a apoptose das células dutais acinares e tubulares, e também por provocar danos no tecido glandular, especialmente pelo linfócito $T^{23}$

$O$ vírus da hepatite $C$ (vírus EpsteinBarr), do herpes simples e outros retrovírus também têm sido associados à etiopatogênese da síndrome de Sjögren primária e às suas complicações, mas sem nenhuma relação definitiva estabelecida. Marcos et al. ${ }^{24}$ encontraram uma prevalência de $0,83 \%$ de infecção crônica por HBV associado à SS; segundo eles, semelhante à prevalência na população geral espanhola $(0,7 \%)$.

\section{Critérios diagnósticos}

Os critérios de classificação e diagnósticos para a SS foram desenvolvidos e validados entre 1989 e 1996 pelo Grupo de Estudos Europeus, ${ }^{25}$ em um estudo multicêntrico realizado em 12 países. Esses abrangeram: sintomas oculares e diminuição do lacrimejamento, sintomas orais e diminuição da salivação, sinais de comprometimento ocular (teste de Schrimer $\mathrm{I}<$ ou $+5 \mathrm{~mm}$ em 5 minutos, Rosa Bengala $>$ ou $=$ a 4), histopatologia (um ou mais focos - aglomerado de 50 ou mais células inflamatórias - em biópsia de glândula salivar menor), envolvimento de glândula salivar (cintilografia, sialografia de parótida, sialometria com fluxo salivar não estimulado < ou = a 1,5 ml em 15 minutos) e anticorpos antiRo (SS-A) ou anti-La (SS-B). ${ }^{26}$

A partir de então, estabeleceram-se seis critérios parâmetros de diagnóstico para essa síndrome: (1) xeroftalmia; (2) xerostomia; (3) exame oftalmológico anormal (teste de Schirmer com resultado inferior $1 \mathrm{~mm} / \mathrm{min}$ ); (4) biópsia de glândula salivar menor evidenciando valores superiores a 1 foco/4 mm; (5) fluxo salivar não estimulado menor do que $1,5 \mathrm{ml} /$ 5 min (exceto para pessoas acima de 60 anos ou em uso de medicamentos anticolinérgicos); e (6) presença dos anticorpos anti-Ro ou anti-La no sangue. ${ }^{27}$ Para SS primária, são necessários quatro critérios positivos entre os seis, enquanto que para a secundária, apenas três critérios específicos são suficientes. ${ }^{28}$

Como procedimento de diagnóstico, inicialmente deve ser verificada a diminuição da função glandular pelo teste de Schirmer e/ou teste saxônica. Após confirmada a SS, segue-se com a detecção de autoanticorpos para Ro (SSA), La (SS-B) e/ou biópsia de uma glândula salivar. ${ }^{29}$

Ressalta-se que a biópsia das glândulas salivares menores do interior do lábio inferior é considerada o teste mais eficaz e definitivo, tendo como resposta positiva a presença de um ou mais agregados de células mononucleares de 50 ou mais células por $4 \mathrm{~mm}^{2}$. O teste laboratorial também pode ser usado e acusa, em alguns pacientes, velocidade de hemossedimentação elevada, hipergamaglobulinemia policlonal e fator reumatoide. Como os métodos de diagnósticos empregados apresentam caráter invasivo, pesquisas vêm sendo realizadas nos últimos anos a fim de desenvolver métodos não invasivos para tal, especialmente pela dosagem de substâncias presentes no processo inflamatório na saliva e nas lágrimas. Contudo, ainda não existem estudos definitivos que validem seu uso como critérios de diagnóstico. ${ }^{30}$

Exames de imagem, como a sialografia por ressonância magnética, têm sido estudados como alternativa não invasiva à sialografia tradicional na tentativa de evitar complicações possíveis, como: trauma do ducto, dor à injeção do contraste, infecção e reação alérgica, e também como alternativa às biópsias. $\mathrm{O}$ estabelecimento do correto diagnóstico da SS 
é fundamental para a instituição do tratamento precoce, que consiste no alívio dos sinais e sintomas, no sentido de modificar o curso da doença, a fim de que as sequelas sejam evitadas ou minimizadas, com repercussões na qualidade de vida dos pacientes. ${ }^{31}$

\section{Tratamento}

Quando estabelecido o diagnóstico, pode-se lançar mão de quatro grupos de fármacos (de acordo com seu princípio ativo) para estabelecer a terapêutica medicamentosa adequada, a saber: repositores tópicos ou orais, imunomoduladores, imunossupressores e biológicos.

Atualmente são utilizados produtos tópicos, como lágrima e saliva, para auxiliar o alívio dos sintomas de secura e a preservação da integridade da córnea, dos dentes e da gengiva. Ademais, tem-se o cloridrato de pilocarpina, o qual estimula a secreção salivar residual em alguns pacientes; também o estrogênio local pode ser utilizado para a secura da pele genital, bem como pomadas para a pele seca. O interferon A (IFNa) surge como outra forma de estimular a produção de saliva e lágrimas, com administração de doses baixas via oral. ${ }^{32}$

Uma alternativa de tratamento está representada pela terapia de empobrecimento de células $B$ malignas com rituximab. Este promove a depleção das células CD20+ e induzindo a apoptose. Atualmente não há recomendação para o uso de fator de necrose tumoral (TNF) a-bloqueio na síndrome de Sjögren. ${ }^{33}$

Fialho et al. ${ }^{34}$ sugerem o uso do ácido micofenólico (MPA), por ser um inibidor seletivo da inosina monofosfato desidrogenase (inibição da via da síntese de nucleotídeos, afetando principalmente linfócitos T e B ativados).

Para o tratamento da ceratoconjuntivite, a terapia inicial consiste na administração de lágrimas artificiais, ou ainda podem ser feitas tentativas de conservar uma película de lágrima por meio da utilização de óculos fechados. Em pacientes com resposta inadequada às lágrimas artificiais, é indicado o fechamento do ponto lacrimal na margem interna das pálpebras, reduzindo assim a drenagem de lágrimas. Trata-se de uma técnica efetiva e simples que não acarreta complicações para o acometido. ${ }^{35}$

Também pode serfeito o uso criterioso de glicocorticoides, AINEs e imunossupressores. O paciente deve evitar ambientes com vento e com pouca umidade, o fumo e as drogas com efeito anticolinérgico. ${ }^{36}$

Outra perspectiva recente no tratamento do olho seco é o uso oral do óleo de linhaça ( $1 \mathrm{~g}$ ou $2 \mathrm{~g}$ ao dia) por possuir em sua composição ácidos graxos ômega 6 e 3. Relatase a melhora dos sintomas e das condições das células epiteliais da conjuntiva, além de uma redução significativa da inflamação da superfície ocular. ${ }^{37}$

O tratamento dos sintomas orais consiste no uso de gomas de mascar ou balas que estimulem a salivação, prevenção de substâncias irritantes como o café, álcool, nicotina, ${ }^{38}$ além de manter uma boa higiene oral e fazer uso tópico de flúor para evitar cáries. Infecções de cândida são frequentes e necessitam de rápida identificação e tratamento. ${ }^{39}$ A pilocarpina e a cevimelina são sialagogos sistêmicos aprovados pelo FDA para o tratamento da xerostomia. ${ }^{40}$

Recomenda-se o uso da clorexidina, associada aos vernizes com flúor, para o controle da placa bacteriana em pacientes com alto risco de cárie dentária e doença periodontal, mesmo na falta de melhores evidências científicas sobre a possível associação entre a SS e essas enfermidades ou na possibilidade de uma higiene oral reforçada pelos indivíduos portadores. ${ }^{41}$ 


\section{Considerações FINAIS}

A SS continua sendo um grande desafio para os profissionais envolvidos na sua abordagem em virtude da imprecisão dos métodos de diagnósticos e etiologia, assim como no tratamento, que consiste apenas no alívio da sintomatologia.

\section{REFERÊNCIAS BIBLIOGRÁFICAS}

1. Rabello LP, Aragão REM, Holanda Filha JG, Ribeiro EM, Dias DR, Rabello LP. Síndrome Sjögren-Larsson. Rev Bras Oftalmol. 2011; 70(6): 416-8.

2. Gomes RS, Raymundi SD, Chahade WH. Síndrome de Sjögren. Temas de Reumatologia Clínica. 2008; 9(1): 45-9.

3. Lima Junior JF, Sena MF, Ferreira MA. Condição oral dos pacientes com síndrome de Sjögren: uma revisão sistemática. Revista Odonto Ciência. 2005; 20(49): 210-16.

4. Amenábar JM, Martins GB, Cherubini K. Síndrome de Sjögren: uma abordagem estomatológica R Ci Méd Biol. 2004; 3(1): 124-9.

5. Neville BW, Damm DD, Allen CM, Bouquot JE. Patologia das glândulas salivares. In: Neville BW, editor. Patología oral e maxilofacial. $3^{\mathrm{a}}$ ed. Rio de Janeiro: Guanabara Koogan; 2009.

6. Bezerra TP, Neto ICP, Dias EOS, Gomes ACA. Síndrome de Sjögren secundária. Arq Odontol. 2010; 46(4): 240-6.

7. Alencar C, Rivaldo EG, Padilha DMP, Frasca LCF. Síndrome de Sjögren: relato de caso. Scientia Medica. 2007; 17(2): 97-100.

8. Oliveira MA, Rezende NP, Maia CM, Gallottini M. Primary Sjögren syndrome in a 2-year-old patient: role of the dentist in diagnosis and dental management with a 6-year follow-up. Int $\mathrm{J}$ Paediatr Dent. 2011; 21(6): 471-5.

9. Fox PC, Bowman SJ, Segal B, Vivino FB, Murukutla N, Choueri K, Ogale S, McLean L. Oral involvement in primary Sjögren syndrome. J Am Dent Assoc. 2008; 139(12): 1592-1601.

10. lacopino AM. Sjögren syndrome: reduced quality of life as an oral-systemic consequence. J Can Dent Assoc. 2010; 76: a98.
11. Laine M, Porola P, Udby L, Kjeldsen L, Cowland JB, Borregaard N, et al. Low salivary dehydroepiandrosterone and androgen-regulated cysteinerich secretory protein 3 levels in Sjögren's syndrome. Arthritis Rheum. 2007; 56(8): 2575-84.

12. Nikolov NP, Illei GG. Patogenesis of Sjögren's syndrome. Curr Opin Rheumatol. 2009; 21(5): 465-70.

13. Båve U, Nordmark G, Lövgren T, Rönnelid J, Cajander S, Eloranta ML, et al. Activation of the type I interferon system in primary Sjögren's syndrome: a possible etiopathogenic mechanism. Arthritis Rheum. 2005; 52(4): 1185-95.

14. Delaleu N, Madureira AC, Immervoll H, Jonsson R. Inhibition of experimental Sjögren's syndrome through immunization with HSP60 and its peptide amino acids 437-460. Arthritis Rheum. 2008; 58(8): 2318-28.

15. Cruvinel WM, Jr DM, Araújo JAP, Salmazi KC, Kállas EG, Andrade LEC. Células T Regulatórias Naturais ( $\left.T_{\text {REGS }}\right)$ em Doenças Reumáticas. Rev Bras Reumatol. 2008; 48(6): 342-55.

16. Sánchez-Guerrero J, Pérez-Dosal MR, Cárdenas-Velázquez F, Pérez-Reguera A, Celis-Aguilar E, Soto Rojas AE, et al. Prevalence of Sjögren's syndrome in ambulatory patients according to the American-European Consensus Group criteria. Rheumatology (Oxford). 2005; 44(2): 235-40.

17. Barbieri R, Chiereghin A. O reumatologista revisita a síndrome de Sjögren. Temas Reumatol Clín. 2009; 10(3): 88-93.

18. Fox RI, Fox CM. Sjögren's syndrome: Infections that may play a role in pathogenesis, mimic the disease, or complicate the patient's course. Indian J Rheumatol. 2011; 6(1): 13-25.

19. Mendes EGP, Ferreira APB, Fernandes SVGS, Rego SL, Cavalcante AC, Simonetti AC. Síndrome de Sjögren: revisão de literatura. Cad Pesq saúde. 2007; 1(2): 37-41.

20. Gomes RS, Brandalise S, Alba GP, Flato UA, Moura Júnior JE. Síndrome de Sjögren primária. Rev Bras Clin Med. 2010; 8(3): 254-65.

21. Voulgarelis MG, Tzioufas A. Pathogenetic mechanisms in the initiation and perpetuation of Sjögren's syndrome. Nat Rev Reumatol. 2010; 6(9): 529-37. 
22. Chiereghin RA. Síndrome de Sjögren. Temas de Reumatol clínica. 2009; 10(3): 21-7.

23. Scagliusi P, D'Amore M, D'Amore S, Scagliusi A. Sjögren's syndrome: apoptosis by anti-SSA and anti-SSB antibodies. Reumatismo. 2006; 58(2): 165-6.

24. Marcos M, Alvarez F, Brito P-Zerón, Bove A, Perez-De-Lis M, Diaz-Lagares C, et al. Chronic hepatitis B virus infection in Sjögren's syndrome. Prevalence and clinical significance in 603 patients. Autoimmun Rev. 2009; 8(7): 616-20.

25. Liquidato BM, Bussoloti Filho I, Camargo ACK, Soler RC. Aspectos do diagnóstico na síndrome de Sjögren. Rev Bras Otorrinolaringol. 2002; 68 (3): 363-7.

26. Felberg S, Dantas PEC. Diagnóstico e tratamento da síndrome de Sjögren. Arq Bras oftalmol. 2006; 69 (6): 959-63.

27. Vitali C. Classification criteria for Sjögren's syndrome. Ann. Rheum. Dis. 2003; 62(1): 94-5.

28. Liquidato BM, Bussoloti Filho I. Avaliação da sialometria e biópsia de glândula salivar menor na classificação de pacientes com Síndrome de Sjögren. Rev. Bras. Otorrinolaringol. 2005; 71(3): 346-54.

29. Witte T. Pathogenese und Diagnostik des Sjögren-Syndroms. Z Rheumatol. 2010; 69 (1): 50-6.

30. Jonsson R, Brun JG. Sjögren's syndrome. Encyclopedia of life sciences (ELS). Chichester: John Wiley \& Sons, 2010.

31. Miedany YME, Ahmed I, Mourad HG, Mehanna AN, Aty SA, Gamal HM, et al. Quantitative ultrasonography and magnetic resonance imaging of the parotid gland: can they replace the histopathologic studies in patients with Sjögren's syndrome? Joint Bone Spine. 2004; 71(1): 29-38.

32. Thanou-Stavraki A, James JA. Primary Sjögren's syndrome: current and prospective therapies. Semin Arthritis Rheum. 2008; 37 (5):273-92.
33. Fassi D, Nielsen $\mathrm{CH}$, Hasselbalch $\mathrm{HC}$, Hegedus $\mathrm{L}$. The rationale for blymphocyte depletion in grace'disease. Monoclonal anti-CD20 antibody therapy as a novel treatment option (Review). Eur J Endocrinol. 2006; 154(5): 623-32.

34. Fialho SCMS, Bergamaschi S, Neves FS, Zimmermann AF, Castro GRW, Pereira IA. Micofenolato mofetil na síndrome de Sjögren primária: uma opção para o tratamento da agranulocitose. Rev Bras Reumatol. 2012; 52(2): 295-299.

35. Forno E, Buzalaf F. Exérese do segmento vertical do canalículo lacrimal nasíndrome do olho seco: estudo preliminar. Arq Bras Oftalmol. 2005; 68(2): 199-203.

36. Feist E, Dörner T, Hansen A. Indikation und Option neuer immunmodulatorischer Therapien beim Sjögren-Syndrom. Z Rheumatol. 2007; 66(8): 679-85.

37. Pinheiro Jr. MN, Santos PM, Santos RCR, Barros JN, Passos LF, Neto JC. Uso oral do óleo de linhaça (linum usitatissimum) no tratamento do olho seco de pacientes portadores da síndrome de Sjögren. Arq Bras Oftalmol. 2007; 70(4): 649-55.

38. Wada K, Niida M, Tanaka M, Kamitani T. Ro52mediated monoubiqui-tination of IKK\{beta\} down-regulates NF-\{kappa\}B signalling. J Biochem. 2009; 146 (6): 821-32.

39. Kassan SS, Moutsopoulos HM. Clinical manifestations and early diagnosis of Sjögren syndrome. Arch Intern Med. 2004; 164(12): 1275-84.

40. Wu CH, Hsieh SC, Lee KL, Li KJ, Lu MC, Yu CL. Pilocarpine hydrochloride for the treatment of xerostomia in patients with Sjögren's Syndrome in Taiwan. A double-blind, placebo-controlled trial. J Formos Med Assoc. 2006; 105 (10): 796-803.

41. DiGangi P, Bendit J. It's $80-20$ with biofilm: update on oral rinses. Dent Today. 2012; 31(3): 58-63.

Submetido em: 21-3-2013

Aceito em: 12-4-2013 